PROCEEDINGS OF THE

AMERICAN MATHEMATICAL SOCIETY

Volume 134, Number 9, September 2006, Pages 2771-2777

S 0002-9939(06)08539-X

Article electronically published on March 23, 2006

\title{
A QUASIFIBRATION OF SPACES OF POSITIVE SCALAR CURVATURE METRICS
}

\author{
VLADISLAV CHERNYSH
}

(Communicated by Alexander N. Dranishnikov)

\begin{abstract}
In this paper we show that for Riemannian manifolds with boundary the natural restriction map is a quasifibration between spaces of metrics of positive scalar curvature. We apply this result to study homotopy properties of spaces of such metrics on manifolds with boundary.
\end{abstract}

\section{INTRODUCTION}

The purpose of this note is to establish the fact that a natural restriction map in Riemannian geometry is a quasifibration of metrics of positive scalar curvature and discuss some applications to the study of spaces of positive scalar curvature metrics.

If $M$ is an open manifold, then, by a result of Gromov Gro69, there always exists on $M$ a metric of positive sectional curvature. However, such a metric, in general, will not be complete. So, when studying metrics of positive scalar curvature on compact manifolds with boundary, it is necessary to impose some sort of a boundary condition. It is natural to require that a metric is a product near the boundary.

Let $M$ be a compact manifold with the boundary $\partial M$. We fix a collar $c: \partial M \times$ $(-1,0] \rightarrow M$ and define $\mathcal{R}^{+}(M)$ to be a space $\{g\}$ of metrics of positive scalar curvature on $M$ such that $c^{*}(g)=g_{0}+d t^{2}$ on $\partial M \times[-1 / 4,0]$. We take the usual Fréchet topology on this space. This topology is defined by the collection of $C^{k}$ norms $\|.\|_{k}$ on the space of all Riemannian metrics $\mathcal{R}\left(M^{n}\right)$ with respect to some reference metric $h:\|g\|_{k}=\max _{i \leq k} \sup _{M^{n}}\left|\nabla^{i} g\right|$. The topology does not depend on a choice of the metric $h$.

With this topology $\mathcal{R}^{+}(M)$ is a Fréchet manifold modeled on the space of symmetric bilinear forms that vanish identically on $c(\partial M \times[-1 / 4,0])$.

By $\mathcal{R}_{0}^{+}(\partial M)$ we denote the image of $\mathcal{R}^{+}(M)$ under the restriction map

$$
\begin{gathered}
\rho: \mathcal{R}^{+}(M) \rightarrow \mathcal{R}^{+}(\partial M), \\
\rho(g):=\left.g\right|_{\partial M},
\end{gathered}
$$

together with the induced topology. We assume that $\mathcal{R}^{+}(M)$ is nonempty, which, of course, implies that $\mathcal{R}_{0}^{+}(\partial M)$ is nonempty. Since $\mathcal{R}^{+}(\partial M)$ is locally convex, the

Received by the editors May 23, 2004 and, in revised form, April 14, 2005.

2000 Mathematics Subject Classification. Primary 58D17, 57R65.

(C)2006 American Mathematical Society 
space $\mathcal{R}_{0}^{+}(\partial M)$ is a union of open path-connected components. This can easily be seen by attaching a linear concordance to the boundary of $M$.

The definition of a quasifibration is due to Dold and Thom DT58. A surjective map $p: E \rightarrow B$ is a quasifibration if for all $x \in B$, all $y \in p^{-1}(x)$, and all $i \geq 0$ we have $\pi_{i}\left(E, p^{-1}(x), y\right) \approx \pi_{i}(B, x)$.

Theorem 1.1. The map $\rho: \mathcal{R}^{+}(M) \rightarrow \mathcal{R}_{0}^{+}(\partial M)$ is a quasifibration.

To show that a map between topological spaces $f: X \rightarrow Y$ is a quasifibration, it suffices to show that its fiber $f^{-1}\left(y_{0}\right)$ is homotopy equivalent to its homotopy fiber under the canonical inclusion map. The homotopy fiber of $f$ at $y_{0} \in Y$ is defined by replacing $f$ by the Serre path fibration $\hat{f}: \hat{X} \rightarrow Y$ and taking the fiber $\Omega_{y_{0}}:=\hat{f}^{-1}\left(y_{0}\right)$. Then $\Omega_{y_{0}}=\{(x, \omega)\}$, where a path $\omega:[0,1] \rightarrow Y$ is such that $\omega(0)=f(x)$ and $\omega(1)=y_{0}$.

The proof of Theorem 1.1 follows from Lemmas 2.2 and 2.3 We introduce an intermediate space $\Omega^{s}$, which is defined by taking smooth paths $\omega$ in the definition of $\Omega_{y_{0}}$. Then we show that $\Omega^{s}$ is homotopy equivalent both to the fiber of $\rho$ and to the homotopy fiber of $\rho$. This implies that the homotopy fiber and the fiber of $\rho$ are homotopy equivalent, and thus completes the proof of Theorem 1.1 .

One of the important geometric implications of Theorem 1.1 is Theorem 1.2 .

A Hausdorff space $X$ is a topological manifold in the sense of Palais Pal66] if there exists an open covering $\left\{O_{\alpha}\right\}$ of $X$ and a family of maps $\left\{\theta_{\alpha}: O_{\alpha} \rightarrow\right.$ $\left.V_{\alpha}\right\}$, where each $V_{\alpha}$ is a locally convex topological vector space and each $\theta_{\alpha}$ is a homeomorphism of $O_{\alpha}$ onto either an open subset of $V_{\alpha}$ or an open subset of a half space of $V_{\alpha}$.

Theorem 1.2. Let $A$ be a contractible subset of $\mathcal{R}_{0}^{+}(\partial M)$. Suppose that $A$ is a metrizable topological manifold. Then for any point $a \in A$ the inclusion $i: \rho^{-1}(a) \rightarrow$ $\rho^{-1}(A)$ is a homotopy equivalence.

Remark 1.1. The conclusion of the above theorem is also true under the assumption that $\rho^{-1}(A)$ is an ANR or, more generally, is dominated by a $C W$-complex. The author does not know whether these properties follow from $A$ merely being contractible.

In particular, if $h_{0}$ and $h_{1}$ are in the same path-connected component of $\mathcal{R}_{0}^{+}(\partial M)$, then the spaces of positive scalar curvature metrics that near the boundary restrict to a product with correspondingly $h_{0}$ and $h_{1}$ are homotopy equivalent.

Another geometric consequence of Theorem 1.1] is an extension of results in Che] to manifolds with boundary.

Let $N^{n-k} \subset M^{n}, k \geq 3$, be a submanifold of $M^{n}$. We assume that there exists a tubular neighborhood $\tau: N \times D^{k} \rightarrow M$, such that the restriction $\tau: \partial N \times D^{k} \rightarrow \partial M$ is a tubular neighborhood of $\partial N$ in $\partial M$. We also assume that the collar $c$ is compatible with $N$ in the sense that its restriction to $\partial N \times(-1,0]$ is a collar for $\partial N$.

We fix a metric $g_{N}$ on $N$ and a torpedo metric $g_{0}$ on $D^{k}$ (a torpedo metric in the $\operatorname{disc} D^{k}$ is an $O(k)$-symmetric, positive scalar curvature metric, which is equal to a $k$-sphere metric near the center of the disc and is a product with a $(k-1)$-sphere metric near the boundary of the disc), such that the metric $g_{N}+g_{0}$ has positive scalar curvature on $N \times D^{k}$. Here the fixed metric $g_{N}$ can be any metric subject to the only requirement that it is a product near the boundary $\partial N$. 
Let $h_{0} \in \mathcal{R}_{0}^{+}(\partial M)$. Since codimension of $\partial N$ in $\partial M$ is greater than 2, from Che we may assume that $\tau^{*}\left(h_{0}\right)=\left.g_{N}\right|_{\partial N}+g_{0}$. We define

$$
\left(\rho^{-1}\left(h_{0}\right)\right)_{0}:=\left\{g \in \rho^{-1}\left(h_{0}\right) \mid \tau^{*}(g)=g_{N}+g_{0}\right\} .
$$

Theorem 1.3. Suppose that $\mathcal{R}^{+}(M)$ is not empty. Then the inclusion map

$$
i:\left(\rho^{-1}\left(h_{0}\right)\right)_{0} \rightarrow \rho^{-1}\left(h_{0}\right)
$$

is a homotopy equivalence.

Example 1.1. Let $M^{n}$ be a manifold with a $k$-handle $D^{n-k} \times D^{k}$, such that $k \geq 3$ and $\mathcal{R}^{+}\left(M^{n}\right)$ is nonempty. Let $g_{1}$ be a metric (which is a product near the boundary) on $D^{n-k}$ and $g_{0}$ a torpedo metric on $D^{k}$, such that $g:=g_{1}+g_{0}$ has positive scalar curvature. Then for any $h_{0} \in \mathcal{R}_{0}^{+}(\partial M)$ the space $\rho^{-1}\left(h_{0}\right)$ is homotopy equivalent to the subspace of $\rho^{-1}\left(\hat{h}_{0}\right)$ consisting of metrics that restrict to the metric $g$ on the handle. Here the metric $\hat{h}_{0}$ is obtained by deforming $h_{0}$ to be equal to $\left.g_{1}\right|_{S^{n-k-1}}+g_{0}$ on $S^{n-k-1} \times D^{k} \subset \partial M$; see Che for details.

\section{Proofs of the theorems}

Given a smooth path of metrics $\alpha: I \rightarrow \mathcal{R}^{+}(X)$ on a closed smooth manifold $X$, we would like to put a positive scalar curvature metric on $X \times \mathbf{R}$. In general, the scalar curvature of the obvious metric $g(x, t)=\alpha(t)(x)+d t^{2}$ will not be positive. However, the metric $\alpha(t)(x)+a d t^{2}$ will have positive scalar curvature for all large enough $a>0$. This is a well-known 'concordance lemma' of Gromov and Lawson; see GL80, Gaj87.

We show that this concordance can be made to depend continuously on the path $\alpha$. Here, the topology on the space of paths is the $C^{\infty}$-topology.

We fix a smooth function $F: \mathbf{R} \rightarrow[0,1]$ such that $0 \leq F^{\prime}<2, F(t)=0, t \in$ $(-\infty, \epsilon], F(t)=1, t \in[1-\epsilon, \infty)$, for some $0<\epsilon<1 / 4$, and for a positive number $\tau$ we define a function $F_{\tau}: \mathbf{R} \rightarrow \mathbf{R}$, by $F_{\tau}(t)=F\left(\frac{t}{\tau}\right)$.

Let

$$
g_{\tau}^{\alpha}(x, t):=\alpha\left(F_{\tau}(t)\right)(x)+d t^{2} .
$$

Define

$$
\begin{aligned}
S^{\prime}(\alpha) & :=\inf _{t>0}\left\{g_{\tau}^{\alpha} \text { is a psc metric for all } \tau \geq t\right\}, \\
S(\alpha) & :=\max \left(S^{\prime}(\alpha), 1\right) .
\end{aligned}
$$

The function $S^{\prime}: \alpha \mapsto S^{\prime}(\alpha)$ is upper semi-continuous. Upper semi-continuity (lower semi-continuity) means that we take a topology on $\mathbf{R}$ generated by the family $\{(-\infty, a)\}$ (correspondingly $\{(a, \infty)\}$ ). By Lemma 2.1 below, it is also lower semi-continuous. It follows that $S$ is a continuous function of a path.

We define a metric on $X \times \mathbf{R}$ by the formula

$$
g_{t_{0}}^{\alpha}(x, t):=\alpha\left(F_{t_{0}}(t)\right)(x)+d t^{2},
$$

where $t_{0}:=S(\alpha)$. From the discussion above, this metric depends continuously on the path $\alpha$. By Lemma 2.1, it has positive scalar curvature and is a Riemannian product near $X \times 0$ and $X \times t_{0}$. 
Lemma 2.1. Let $\alpha:[0,1] \rightarrow \mathcal{R}^{+}\left(X^{n}\right)$ be a $C^{\infty}$-family of positive scalar curvature metrics on a compact closed manifold $X^{n}$. Then:

(i) $\exists \lambda>0$ such that $g^{\lambda}(t):=\alpha\left(F_{\lambda}(t)\right)+d t^{2} \in \mathcal{R}^{+}\left(X^{n} \times[0, \lambda]\right)$ and $g^{\lambda}$ is a product metric near the boundary $(X \times 0) \cup(X \times 1)$ of $X \times[0, \lambda]$;

(ii) if $t_{0}=S^{\prime}(\alpha)$ is a positive number, then $\forall m=1,2,3, \ldots \exists t_{m}>0, x_{m} \in$ $X^{n}, \tau_{m} \in\left[0, t_{m}\right]$ such that $t_{0}-\frac{1}{m}<t_{m}<t_{0}$ and the scalar curvature of $\alpha\left(F_{t_{m}}(t)\right)+d t^{2}$ at $\left(x_{m}, \tau_{m}\right)$ is negative.

Proof. (i) Denote $g^{\lambda}(x, t):=\alpha\left(F_{\lambda}(t)\right)(x)+d t^{2}$. Let $\left(x_{0}, \tau_{0}\right)$ be a point in $X^{n} \times[0, \lambda]$. Take normal coordinates for $\alpha\left(F_{\lambda}\left(\tau_{0}\right)\right)$ at a point $x_{0} \in X^{n}$. In these coordinates, we get $g_{i j}^{\lambda}\left(x_{0}, \tau_{0}\right)=\delta_{i j}, \Gamma_{i j}^{k}=0$ for $1 \leq i, j, k \leq n$. Recall that

$$
\Gamma_{i j}^{k}=\frac{1}{2} g^{k l}\left(\partial_{i} g_{j l}+\partial_{j} g_{i l}-\partial_{l} g_{i j}\right) .
$$

Since $g_{i, n+1}^{\lambda} \equiv 0$ for $1 \leq i \leq n$, we get in our normal coordinates at $\left(x_{0}, \tau_{0}\right)$ :

$$
\begin{aligned}
\Gamma_{i j}^{k}\left(x_{0}, \tau_{0}\right) & =0 \quad \text { for } 1 \leq i, j, k \leq n, \\
\Gamma_{n+1, i}^{n+1} & \equiv 0 \quad \text { for } 1 \leq i \leq n+1, \\
\Gamma_{n+1, j}^{i}\left(x_{0}, \tau_{0}\right) & =\frac{1}{2} \partial_{n+1} g_{i j}\left(x_{0}, \tau_{0}\right) \quad \text { for } 1 \leq i, j \leq n, \\
\Gamma_{i j}^{n+1}\left(x_{0}, \tau_{0}\right) & =-\frac{1}{2} \partial_{n+1} g_{i j}\left(x_{0}, \tau_{0}\right) \quad \text { for } 1 \leq i, j \leq n,
\end{aligned}
$$

and the equations for sectional curvatures are

$$
\begin{aligned}
R_{i j k}^{s} & =\partial_{j} \Gamma_{i k}^{s}-\partial_{i} \Gamma_{j k}^{s}+\Gamma_{i k}^{l} \Gamma_{j l}^{s}-\Gamma_{j k}^{l} \Gamma_{i l}^{s}, \\
R_{i j k s} & =R_{i j k}^{l} g_{l s}, \\
K_{i j} & =\left(\partial_{i}, \partial_{j}, \partial_{i}, \partial_{j}\right)=R_{i j i j} .
\end{aligned}
$$

From the Gauss equation for the curvature we get for $1 \leq i, j \leq n$

$$
K_{i j}=\bar{K}_{i j}+\left(b_{i i} b_{j j}-b_{i j}^{2}\right) .
$$

The remaining sectional curvatures are

$$
\begin{aligned}
K_{i, n+1} & =R_{i, n+1, i}^{n+1} \\
& =\partial_{n+1} \Gamma_{i i}^{n+1}-\partial_{i} \Gamma_{n+1, i}^{n+1}+\Gamma_{i i}^{l} \Gamma_{n+1, l}^{n+1}-\Gamma_{n+1, i}^{l} \Gamma_{i l}^{n+1} \\
& =\partial_{n+1} \Gamma_{i i}^{n+1}-\Gamma_{n+1, i}^{l} \Gamma_{i l}^{n+1} \\
& =-\frac{1}{2} \partial_{n+1}^{2} g_{i i}-\left(\frac{1}{2} \partial_{n+1} g_{l i}\right)\left(-\frac{1}{2} \partial_{n+1} g_{i l}\right) \\
& =-\frac{1}{2} \partial_{n+1}^{2} g_{i i}+\frac{1}{4} \sum_{l=1}^{n+1}\left(\partial_{n+1} g_{i l}\right)^{2} .
\end{aligned}
$$

Then the scalar curvature at a point $\left(x_{0}, \tau_{0}\right)$ is given by the formula

$$
\begin{aligned}
\kappa=\kappa_{X} & +\sum_{i, j=1}^{n}\left(b_{i j}^{2}-b_{i i} b_{j j}\right) \\
& -\sum_{i=1}^{n} \partial_{n+1}^{2} g_{i i}+\frac{1}{2} \sum_{i, j=1}^{n}\left(\partial_{n+1} g_{i j}\right)^{2} .
\end{aligned}
$$


Now, we have that $b_{i j}=\Gamma_{i j}^{n+1}=-\frac{1}{2} \partial_{n+1} g_{i j}\left(x_{0}, \tau_{0}\right)$ and the formulas for the derivatives

$$
\begin{aligned}
\partial_{n+1} g_{i j}\left(x_{0}, \tau_{0}\right) & =\frac{1}{t_{0}} F^{\prime}\left(\tau_{0}\right) \alpha^{\prime}\left(F\left(\frac{\tau_{0}}{t_{0}}\right)\right)_{i j}\left(x_{0}\right), \\
\partial_{n+1}^{2} g_{i j}\left(x_{0}, \tau_{0}\right) & =\frac{1}{t_{0}^{2}} F^{\prime \prime}\left(\tau_{0}\right) \alpha^{\prime}\left(F\left(\frac{\tau_{0}}{t_{0}}\right)\right)_{i j}\left(x_{0}\right) \\
& +\frac{1}{t_{0}^{2}}\left(F^{\prime}\left(\tau_{0}\right)\right)^{2} \alpha^{\prime \prime}\left(F\left(\frac{\tau_{0}}{t_{0}}\right)\right)_{i j}\left(x_{0}\right) .
\end{aligned}
$$

The scalar curvature for the product may now be expressed as

$$
\begin{aligned}
\kappa=\kappa_{X} & +\frac{1}{4} \frac{1}{t_{0}^{2}} \sum_{i, j=1}^{n}\left(F^{\prime}\left(\frac{\tau_{0}}{t_{0}}\right)\right)^{2}\left(\left(\alpha_{i j}^{\prime}\right)^{2}-\alpha_{i i}^{\prime} \alpha_{j j}^{\prime}\right)\left(x_{0}\right) \\
& +\frac{1}{t_{0}^{2}} \sum_{i=1}^{n}\left(F^{\prime \prime}\left(\frac{\tau_{0}}{t_{0}}\right) \alpha_{i i}^{\prime}\left(x_{0}\right)+\left(F^{\prime}\left(\frac{\tau_{0}}{t_{0}}\right)\right)^{2} \alpha_{i i}^{\prime \prime}\left(x_{0}\right)\right) \\
& +\frac{1}{2} \frac{1}{t_{0}^{2}} \sum_{i, j=1}^{n}\left(F^{\prime}\left(\frac{\tau_{0}}{t_{0}}\right)\right)^{2}\left(\alpha_{i j}^{\prime}\left(x_{0}\right)\right)^{2} .
\end{aligned}
$$

To finish the proof, note that $\kappa_{X}$ is positive for all $(x, \tau) \in X^{n} \times\left[0, t_{0}\right]$.

(ii) Suppose $t_{0}>0$ and let $\left(x_{0}, \tau_{0}\right)$ be a point in $X^{n} \times\left[0, t_{0}\right]$ where the scalar curvature is not positive. Such a point always exists since the $\kappa>0$ is an open condition, and if the scalar curvature is everywhere positive we can find $t_{1}<t_{0}$ such that the metric corresponding to $t_{1}$ will have positive scalar curvature. Now, freeze the values of $t_{0}, \tau_{0}$, and $x_{0}$ which are in the arguments for the functions $F, \alpha$ and their derivatives, and regard the resulting function as a function of the inverse of $t_{0}$. In light of the argument above it has a positive derivative at $t_{0}$ and its value at $t_{0}$ is less or equal than 0 . So in an arbitrary neighborhood on the left from $t_{0}$ we can find a value $t_{m}$ such that our function will be strictly negative at the point $t_{m}$. Now, "unfreezing" only $\tau_{0}$, we can find a number $\tau_{m}$ such that $\frac{\tau_{m}}{t_{m}}=\frac{\tau_{0}}{t_{0}}$. The point $\left(x_{0}, \tau_{m}\right)$ is the one that we were seeking.

We fix a metric $h_{0} \in \mathcal{R}_{0}^{+}(\partial M)$ and consider the homotopy fiber $\Omega$ of $\rho$ at $h_{0}$, $\Omega=\left\{(g, \omega) \mid \omega(0)=\rho(g), \omega(1)=h_{0}\right\}$. The topology on this fiber is the usual compact-open topology. The smooth homotopy fiber $\Omega^{s}$ is defined analogously by taking $\omega$ to be a smooth path. We take the Fréchet topology on the smooth fiber.

There is a natural embedding $i$ of the fiber $\rho^{-1}\left(h_{0}\right)$ into $\Omega^{s}, i(g)=(g, *)$, where $*$ is the constant path $*(t)=h_{0}$.

Lemma 2.2. The map $i: \rho^{-1}\left(h_{0}\right) \rightarrow \Omega^{s}$ is a homotopy equivalence.

Proof. Let $(g, \omega)$ be a point in $\Omega^{s}$ and let $V_{0}$ be a constant outward normal vector field on $\partial M$ of unit length. We take a smooth cutoff function $\psi$ on $\mathbf{R}$ with $\psi(-3 / 4)=0, \psi(-1 / 4)=1$, and define a vector field on $M$ by setting

$$
V(x)= \begin{cases}\psi(t) S(\omega) V_{0}, & x=c(a, t) \\ 0 & \text { otherwise }\end{cases}
$$


where $S$ is defined by formula (1). Extend this vector field to $M \cup(\partial M \times[0, \infty))$ as a constant vector field $S(\omega) V_{0}$ on $\partial M \times[0, \infty)$ and denote by $\Phi_{1}^{S}$ the diffeomorphism determined by the flow of this vector field at $t=1$. Then $\Phi_{1}^{S}(M)=M \cup$ $(\partial M \times[0, S])$. Define

$$
g^{\omega}= \begin{cases}g & \text { on } M, \\ g_{t_{0}}^{\omega} & \text { on } \partial M \times[0, S], \\ h_{0} & \text { on } \partial M \times[S, \infty),\end{cases}
$$

where $g_{t_{0}}^{\omega}$ is given by the formula (2).

Now, we define an inverse map $r: \Omega^{s} \rightarrow \rho^{-1}\left(h_{0}\right)$ as

$$
r(g, \omega):=\left(\Phi_{1}^{S(\omega)}\right)^{*}\left(g^{\omega}\right) .
$$

Here we take the restriction of the pullback metric to $M$.

For $u \in[0,1]$ we define a path $\omega_{u}(\tau):=\omega((1-u) \tau+u)$. Let $g_{u F}^{\omega}$ be the metric that is defined exactly as $g^{\omega}$ by taking the function $u F$ instead of $F$. The homotopy $H: \Omega^{s} \times[0,1] \rightarrow \Omega^{s}$ of $i \circ r$ to the identity map is given by

$$
H((g, \omega), u)= \begin{cases}\left(\left(\Phi_{1}^{2 u S(\omega)}\right)^{*}\left(g_{0 F}^{\omega}\right), \omega_{0}\right), & 0 \leq u \leq 1 / 2 \\ \left(\left(\Phi_{1}^{S(\omega)}\right)^{*}\left(g_{(2 u-1) F}^{\omega}\right), \omega_{2 u-1}\right), & 1 / 2 \leq u \leq 1\end{cases}
$$

When $u$ is equal to 0 , the map $H(\cdot, 0)$ is the identity map on $\Omega^{s}$. When $u=1$, the map $H(\cdot, 1)$ is equal to $i \circ r$.

Lemma 2.3. The inclusion map $i: \Omega^{s} \rightarrow \Omega$ is a homotopy equivalence.

Proof. The proof is completely analogous to the proof of Theorem 17.1 in Mil63. Since $\Omega$ is an open subset of a locally convex topological vector space, we can cover it with convex open sets. Then take $\Omega_{k}$, the space of all paths $\omega$ such that $\omega\left(\left[(j-1) / 2^{k}, j / 2^{k}\right]\right)$ is contained in some element of the covering. The space $\Omega$ is a homotopy direct limit of $\Omega_{k}$, and the space $\Omega^{s}$ is a homotopy direct limit of $\Omega_{k}^{s}:=i^{-1}\left(\Omega_{k}\right)$. By Milnor's argument, the map

$$
\left.i\right|_{\Omega_{k}^{s}}: \Omega_{k}^{s} \rightarrow \Omega_{k}
$$

is a homotopy equivalence. Here, the inverse map is defined by taking a path $\omega \in \Omega_{k}$ and assigning to it a piecewise linear path that coincides with $\omega$ at points $j / 2^{k}$. Then we smooth the resulting path by pre-composing it with a smooth function that maps $j / 2^{k}$ to $j / 2^{k}$ and all of whose derivatives vanish at points $j / 2^{k}$. This finishes the proof.

Proof of Theorem 1.2. If $p: E \rightarrow B$ is a quasifibration over a contractible space $B$, then for any point $b \in B$ the inclusion of the fiber $p^{-1}(b) \rightarrow E$ induces a weak homotopy equivalence. From Palais [Pal66] we know that both $\rho^{-1}(a)$ and $\rho^{-1}(A)$ are dominated by $C W$-complexes. For such dominated spaces a weak homotopy equivalence is, in fact, a homotopy equivalence by a theorem of J. H. C. Whitehead. From Theorem 1.1 it follows that the inclusion map $i$ is a homotopy equivalence.

Proof of Theorem 1.3. As in the proof of Theorem 1.2, it suffices to show that $i$ is a weak homotopy equivalence. In [Che a method for deforming compact families of metrics of positive scalar curvature was developed, which allowed us to prove the weak homotopy equivalence in the case of closed manifolds $M$ and $N$. This 
deformation can be readily adapted to manifolds with boundary and has an important property. Namely, it preserves the product structure with respect to the fixed tubular map $\tau$, i.e. if $\tau^{*}(g)=g_{N}+g_{0}$, then for the deformation metrics $g(t)$, $t \in[0,1]$, we have $\tau^{*}(g(t))=g_{N}+g_{0}(t)$ and $g(t)$ is constant outside of the tubular neighborhood of $N$. The problem is that, in general, $g_{0}(t)$ is not equal to $g_{0}$, so this deformation takes us outside the fiber $\rho^{-1}\left(h_{0}\right)$.

The solution is to introduce a subspace $A \subset \mathcal{R}_{0}^{+}(\partial M)$ consisting of metrics that are equal to $h_{0}$ outside $\tau\left(\partial N \times D^{k}\right)$ and equal to $\left.g_{N}\right|_{\partial N}+g_{w}$ on $\tau\left(\partial N \times D^{k}\right)$. Here, $g_{w}$ is a warped metric in the disc, i.e. $g_{w}=g(t)^{2} d t^{2}+f(t)^{2} d \xi^{2}$, where $d \xi^{2}$ is the standard metric of the $(k-1)$-sphere of radius $1, g$ is a smooth even function, and $f$ is a smooth odd function. Note that $g_{0} \in A$, i.e. a torpedo metric is a warped metric. Then, from the construction of the deformation, we have that $g_{w}(t)$ is a warped metric for all $t \in[0,1]$. This allows us to conclude a weak homotopy equivalence (and, therefore, a homotopy equivalence) between $\left(\rho^{-1}\left(h_{0}\right)\right)_{0}$ and $\rho^{-1}(A)$.

From [Che] it follows that the inclusion map $h_{0} \rightarrow A$ is a weak deformation retraction; cf. Theorem 4.1 in Che. Now, the proof follows from Theorem 1.2 ,

\section{REFERENCES}

[Che] Vladislav Chernysh, On the homotopy type of the space $\mathcal{R}^{+}(M)$, Preprint, arXiv: math.GT/0405235.

[DT58] Albrecht Dold and René Thom, Quasifaserungen und unendliche symmetrische Produkte, Ann. of Math. (2) 67 (1958), 239-281. MR0097062(20:3542)

[Gaj87] Paweł Gajer, Riemannian metrics of positive scalar curvature on compact manifolds with boundary, Ann. Global Anal. Geom. 5 (1987), no. 3, 179-191. MR0962295 (89m:53061)

[Gro69] M. L. Gromov, Stable mappings of foliations into manifolds, Izv. Akad. Nauk SSSR Ser. Mat. 33 (1969), 707-734. MR.0263103 (41:7708)

[GL80] Mikhael Gromov and H. Blaine Lawson, Jr., The classification of simply connected manifolds of positive scalar curvature, Ann. of Math. (2) 111 (1980), no. 3, 423-434. MR0577131 (81h:53036)

[Mil63] J. Milnor, Morse theory, Based on lecture notes by M. Spivak and R. Wells. Annals of Mathematics Studies, No. 51, Princeton University Press, Princeton, N.J., 1963. MR0163331(29:634)

[Pal66] Richard S. Palais, Homotopy theory of infinite dimensional manifolds, Topology 5 (1966), 1-16. MR0189028 (32:6455)

Mathematisches Institut, Georg-August-Universität Göttingen, Bunsenstrasse 3-5, 37073 Göttingen, Germany

E-mail address: vchernys@uni-math.gwdg.de 